\title{
Usos y apropiaciones de las redes sociales digitales en adultos mayores
}

\author{
Jaime Miguel González Chávez ${ }^{1 *}$, Efraín Delgado Rivera²
}

\begin{abstract}
Resumen
Los estudios de redes sociales digitales generalmente se orientan a comprender las prácticas ejecutadas por jóvenes y adultos, sin embargo, este trabajo se enfoca en adultos mayores. Explora cómo las formas de interacción hipermediada constituyen un factor de exclusión o de empoderamiento en este grupo etario. La pregunta central indaga en los usos y apropiaciones de las redes sociales digitales por parte de los adultos mayores. En este sentido, se trabaja por método etnográfico en comunidades virtuales y por entrevista semiestructurada online y offline, para indagar prácticas y representaciones sociales que se desprenden de esta interacción en usuarios y no-usuarios.

Abstract

Studies of digital social networks are generally oriented to understand the practices carried out by young people and adults, however, this work focuses on seniors. This study explores how hypermediated interaction forms constitute a factor of exclusion or empowerment in this age group. The central question explores the uses and appropriations of digital social networks by seniors. In this sense, we work ethnographically in virtual communities and through semi-structured interviews online and offline, to research practices and social representations that emerge from this interaction in users and non-users.
\end{abstract}

\section{Palabras Clave}

Adultos mayores, Social media, Brecha digital, Apropiación tecnológica

Keywords

Older adults, Social media, Digital divide, Technological appropriation

${ }^{1,2}$ Doctores en Ciencias y Humanidades para el desarrollo interdisciplinario. Universidad De La Salle, Bajío, México

*Autor para correspondencia: jaimegonzalezchavez@gmail.com

\section{Introducción}

Las sociedades contemporáneas ancladas en el mundo industrializado y en vías de desarrollo han incorporado para su funcionamiento toda una estructura tecnológica que sustenta sus actividades más complejas. Desde la actividad macro y micro económica, hasta las más diversas formas de expresión cultural; pasando por el ámbito de las prácticas políticas, hasta las más variadas manifestaciones del mundo del espectáculo y la sociabilidad cotidiana.

Esta relevancia constituye un verdadero motor de la actividad técnico científica en las comunidades que buscan mantenerse a la vanguardia de la amplia gama de dispositivos que soportan sus prácticas. En este contexto, la reflexividad científica y tecnológica se ha ocupado particularmente de aquellos sectores sociales usuarios/consumidores que integran este conjunto de actividades productivas en términos principalmente económicos.

Esta realidad, de fondo utilitarista, ha detonado una disparidad en el acceso y usos asignados a las tecnologías de información y de comunicaciones, ya sea por factores económicos -algunos de carácter distributivo- o por deficiencia en la apropiación tecnológica. Esta situación ha sido ya bien identificada como la "brecha digital" (digital divide), y constituye una de las preocupaciones internacionales de 
acuerdo a la Organización de Naciones Unidas, que estableció en 2003 la Cumbre Mundial de la Sociedad de la Información en Ginebra.

Uno de sus sectores excluidos es el de los adultos mayores, los cuales representan un aspecto complejo de crecientes proporciones, tomando en cuenta, además, que la población mundial se dirige rápidamente a una inversión etaria en donde cada vez más se acrecientan los rangos de 60 a más edad. El mundo envejece, generando problemáticas que incluyen dimensiones como la salud, la economía, el trabajo, la educación y, en general, en la calidad de vida de este sector.

El presente estudio aborda este rango etario y se realizó en la ciudad de León, Guanajuato. Esta área urbana es una de las principales referencias de desarrollo económico en la entidad, la cual ocupa, según Forbes (Villafranco, 2015), el quinto lugar entre las 32 entidades y es León su principal motor. Además, esta misma ciudad, con más de 1 millón 600 mil habitantes, tiene un sector poblacional mayor de sesenta años de 100299 personas, según el más reciente censo de 2010, lo que equivale al $7 \%$ de la población de León (INEGI, 2010).

Esto coloca a León como un referente de caso de estudio tomando en cuenta las características de la media mundial, donde según la Organización Mundial de la Salud, "Entre 2000 y 2050, la proporción de los habitantes del planeta mayores de 60 años se duplicará, pasando del $11 \%$ al 22\%. En números absolutos, este grupo de edad pasará de 605 millones a 2000 millones en el transcurso de medio siglo" (OMS, s.f.).

Particularmente, algunas de las problemáticas detonadas con el envejecimiento de la población en los países desarrollados tienen que ver con la disminución del grupo de personas en edad de trabajar:

a) Demanda poblacional por servicios de salud.

b) Desarrollo de servicios de desarrollo social.

c) Incremento en el pago de pensiones.

d) Necesidad de aumento en la edad de retiro.

En un sentido sintético, el sistema capitalista promueve una representación del envejecimiento donde la persona carece de valor en tanto que es incapaz de producirlo en el sistema económico. Se puede argumentar que en el sistema económico de sociedades modernas se profundiza la experiencia de un mundo diseñado para los sectores productivos. En este sentido, la tecnología constituye una poderosa fuente de exclusión.

En México, la proporción de personas de 55 y más años es del $8 \%$. El uso de internet se observa en la Figura 1, el cual representa el $51 \%$ de la población usuaria.

\section{Usuarios de computadora o internet por grupos de edad, 2017.}

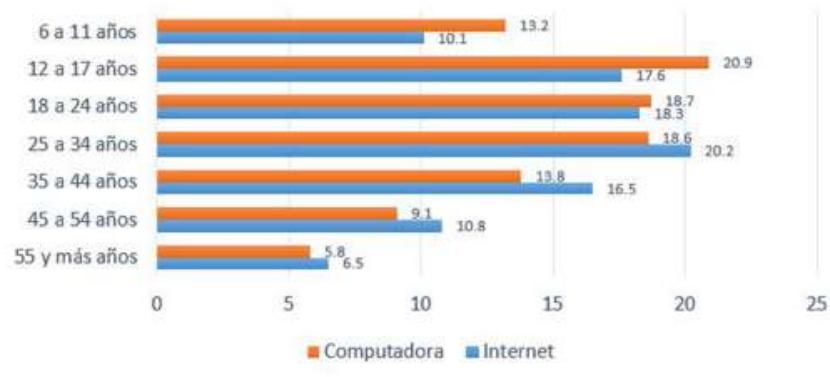

Figura 1. Usuarios de computadora e internet por grupo etario. Fuente: INEGI (2017b)

Es decir, si el $51 \%$ de mexicanos usa internet y de esa población sólo el $6.5 \%$ de usuarios es mayor de 55 años, esto equivale a menos de $25 \%$ de la población de mexicanos adultos mayores.

Sin embargo, existe un incremento de adultos mayores usuarios de internet que refleja la incorporación de estas tecnologías en las dinámicas cotidianas, adquiriendo el lenguaje apropiado para el ciberespacio, habilidades y experiencias hipermediatizadas de ecologías cibernéticas que han sido diseñadas especialmente para jóvenes y usuarios adultos de edad mediana.

Dentro de estas ecologías, las más populares resultan ser las redes sociales digitales, ya que la mayor actividad de usuarios $(88.9 \%)$, según la encuesta de usos de internet de INEGI (2017a, p. 4) es para comunicación, con un $75.6 \%$ en acceso a redes sociales. Estas redes son utilizadas como reforzadores para la consolidación de relaciones afectivas construidas a lo largo del tiempo, por lo que en la vejez se esperaría un importante valor de socialización para el individuo.

En este estudio se explora el uso de estos espacios y tecnologías incorporadas por los adultos mayores para generar una mayor comprensión que permita dilucidar beneficios, así como brechas de exclusión derivadas del 
acceso, alfabetización digital, lenguajes, elevados precios de la tecnología y sus rápidas transformaciones.

\section{Problemáticas y condiciones sociales del adulto mayor}

Uno de los principales factores para medir la calidad de vida de un adulto mayor reside en la densidad de las relaciones afectivas. Es decir, la inclusión social y su participación dentro de su grupo.

Los procesos de exclusión social son tanto culturales como económicos. En una sociedad donde la participación social está centrada en la capacidad de producción, la vejez constituye un sinónimo de incompetencia social, por lo que muchos adultos mayores son percibidos como una carga para el resto, incluso para sus propios familiares, quienes tienen que velar por las necesidades de una persona incapacitada para proveerse de algunas de sus necesidades.

\section{Propuesta metodológica para la observación de prácticas mediadas por internet en adultos mayores}

\subsection{Una mirada etnográfica a usuarios de comunidades virtuales de internet}

Las comunidades virtuales, según Galindo Cáceres (2000) aparecen como grupos de pares que comparten intereses, mismos que convocan a la agrupación. Sin embargo, aunque estas comunidades son anteriores a internet (este autor menciona como ejemplo a las comunidades académicas de hace dos siglos), es precisamente la aparición de esta herramienta la que proporciona un nuevo efecto en la configuración de estos grupos, potenciando su alcance y facilitando las interacciones en tiempo y espacio, es decir, en el ciberespacio.

Para explorarlas, existen procedimientos etnográficos de rápida ejecución, como el Rapid Appraisial Procedures o el Rapid Ethnographic Assessment (DeWalt y DeWalt, 2011). En este caso, la etnografía de situación (usada por investigadores como Marc Augè) remite a la observación de una situación particular, aquí determinada por el acto de interacción de los adultos mayores en espacios virtuales mediados por tecnologías de comunicación.
La operatividad de la metodología no dista mucho de la etnografía presencial (Hine, 2004; DeWalt y DeWalt, 2011), la cual propone un acercamiento por medio de registros que pueden ser parte de notas en diarios de campo. Para la observación virtual se utilizó el programa Evernote, ya que permite "atrapar" pantallas, interactuar con ellas y funcionar como cuaderno de notas (en el sentido tradicional de "diario de campo").

En este contexto, internet se convierte en un espacio que definitivamente construye relaciones e intercambios de sentido en un ir y venir de textos. Aunque autores como Hammersley y Atkinson (1995) centran la reflexión en torno a una etnografía que enfoca su atención en los discursos orales dando un valor secundario a textos escritos, hay que reconocer que los textos digitales son interpretaciones de la realidad por parte de los sujetos en interacción mediada, susceptibles de ser analizados.

En este acercamiento se propone un rol periférico del observador, es decir, una participación moderada (Spradley, 1980). Este tipo de observación tiene el objetivo de permitir la interactividad natural de los miembros de grupos sin intervenir de manera directa en las temáticas ni en los intercambios textuales.

Delimitación temporal: $\mathrm{Se}$ realizan 400 horas de observación.

Delimitación geográfica del usuario: León, Guanajuato. Aunque el recorte espacial del estudio esté centrado en la ciudad de León, es necesario reconocer la inestabilidad de las fronteras en diseños virtuales. Esta característica es más sólida cuando se refiere a la muestra de entrevista.

Entorno de observación/ecología: Interfaz de Facebook.

Situación de observación: "Grupos Especializados" en Facebook.

Actores: Miembros/usuarios mayores de 60 años.

\subsection{Entrevistas semi estructuradas a usuarios y no usuarios de red social en internet}

- Usuarios habituales de la red social digital de Facebook y no usuarios.

- Doce entrevistas totales

Las entrevistas fueron analizadas por el método gramatical argumentativo y temático, de acuerdo a la propuesta de 
Tabla 1. Comunidades virtuales observadas.

\begin{tabular}{|c|c|c|c|c|}
\hline Grupo de Facebook & Descripción & Tipo & Miembros & Observación \\
\hline $\begin{array}{l}\text { Adultos mayores } \\
\text { cibernéticos }\end{array}$ & $\begin{array}{l}\text { "Para todos los mayores de la tercera edad, } \\
\text { simplemente para mayores" }\end{array}$ & Público & 481 & 100 horas \\
\hline $\begin{array}{l}\text { Las abuelas más } \\
\text { felices del mundo }\end{array}$ & $\begin{array}{l}\text { "deben de ser abuelas y ser las mas felices...y sobre } \\
\text { todo difundir el amor a nuestros nietos...y que } \\
\text { somos muy indispensables en el crecimiento del } \\
\text { amor a nosotros.." }\end{array}$ & Público & 2061 & 100 horas \\
\hline Abuelos Gay & $\begin{array}{l}\text { "Bienvenidos todas aquellas personas con criterio } \\
\text { formado y que quieran participar del grupo, } \\
\text { esta prohibido subir desnudos totales, enseñar } \\
\text { partes Íntimas, pues se expone a ser retirado del } \\
\text { grupo....saludos cordiales a todos!" }\end{array}$ & Público & 318 & 100 horas \\
\hline $\begin{array}{l}\text { Abuelos \& } \\
\text { Abuelas } \\
\text { Alcaguetes } \\
\text { (Alcahuetes) }\end{array}$ & $\begin{array}{l}\text { "Demuestra lo orgulloso u orgullosa que estas } \\
\text { con tus nietos, que no te importe que te llamen } \\
\text { alcaguete, apoyador, etc. No tienes que ser abuelo } \\
\text { o abuela para ser miembro de este grupo, tambien } \\
\text { se aceptan los nietos y creo que todos los somos". }\end{array}$ & Público & 144 & 100 horas \\
\hline
\end{tabular}

Fuente: Elaboración propia.

Nota: Se muestran los textos de Facebook tal como fueron publicados.

Tabla 2. Muestra por saturación de entrevista semiestructurada.

\begin{tabular}{|c|c|c|c|}
\hline $\begin{array}{l}\text { Número de } \\
\text { entrevistas }\end{array}$ & Categoría & Edades & Criterios de selección \\
\hline 6 & Usuarios de Facebook & Mayores de 60 años & $\begin{array}{l}\text { Frecuencia de uso de la red mayor a dos } \\
\text { horas al día. Pertenecientes a grupos activos } \\
\text { en Facebook. Habitantes de la ciudad de } \\
\text { León. }\end{array}$ \\
\hline 6 & No Usuarios de Internet & Mayores de 60 años & $\begin{array}{l}\text { Habitantes de la ciudad de León. Ajenos al } \\
\text { uso de internet por decisión propia. }\end{array}$ \\
\hline
\end{tabular}

Fuente: Elaboración propia.

Galindo Cáceres (1992) en el cual se distinguen las categorías analíticas, los temas y las enunciaciones vertidas por los individuos.

\section{Usos y apropiaciones en adultos mayores}

Uno de los hallazgos principales sobre el uso de la red social es que no existe una comunicación efectiva; sin embargo, también provee otros satisfactores discutibles. Esto no significa que sea un aspecto privativo del sector de mayores de sesenta años.

Durante las interacciones se genera un repertorio diverso de acciones comunicativas en donde su expresión es habitualmente por medio de imágenes acciones que no determinan que exista un diálogo o proceso comunicativo. Esta distinción parte de la sociología de la comunicación y su modelo básico (Schützeichel, 2015), que entiende la "acción comunicativa" como un reportorio de selecciones o actos ilocutorios compuestos por textos, imágenes, sonidos, etc., que deberían articularse en la interacción como un "proceso comunicativo" o diálogo, el cual ya incorpora estos "sucesos comunicacionales".

Estas acciones pocas veces generan un proceso comunicativo observable ya que en la mayoría de casos no se consuma la interacción, al carecer de elementos de retroalimentación observables. 


\subsection{La preponderancia de la imagen sobre el discurso}

La comunicación en Facebook resulta más una ilusión colectiva de comunicación, en la cual se comparte información personal que solo da sentido al usuario en el contexto de su propia experiencia.

La Figura 2 muestra el repertorio de acciones comunicativas limitadas al acto share con un $96 \%$ contra un $4 \%$ de intervenciones textuales. Esto es preámbulo de una interacción comunicativa casi nula. Además, dentro de la categoría share se prioriza la imagen (79\%) sobre cualquier otro contenido, tal como se ilustra en la Figura 3.

\section{ACCIONES COMUNICATIVAS}

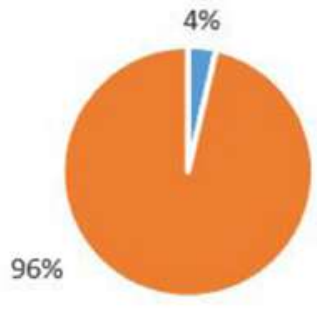

" COMENTARIOS " SHARE

Figura 2. Acciones comunicativas en grupos de adultos mayores en Facebook.

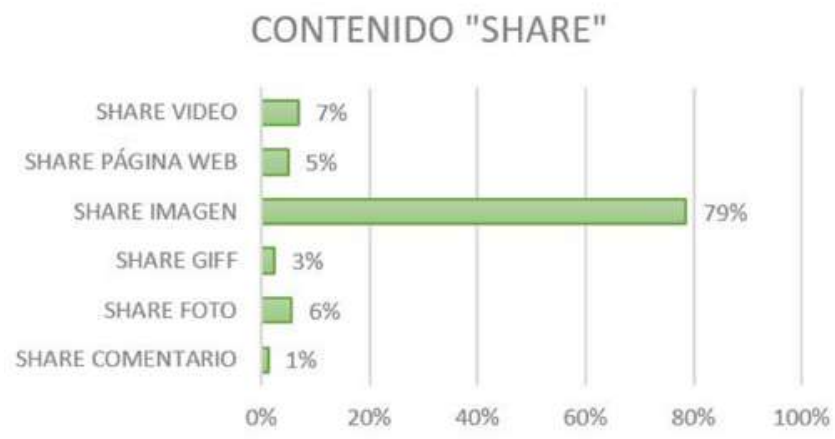

Figura 3. Contenido de acciones share.

\subsection{El uso informativo}

Las redes sociales digitales son usadas para compartir información la cual es atendida por la comunidad de manera superficial o simplemente ignorada. La información atendida funge como un reforzador e identificador de ideas del usuario y del grupo.
Esta información es prioritariamente de contenido personal. Tiene la finalidad de expresar la identidad del ser abuelo en vez de buscar formas de coordinación entre sujetos de una comunidad por algún fin común o alguna discusión de ideas.

En estas expresiones, el papel central es de la figura del "nieto". Esta figura binaria (abuelo-nieto), además de constituir un lazo afectivo fundamental, representa trascendencia personal e identidad en el rol de abuelo. Dicho rol es importante para la autoestima del adulto mayor que encuentra en este una motivación para sus actividades cotidianas. La imagen del "nieto" en la mayor parte de los casos no es explícita, sino que se construye metafóricamente.

\subsection{El contenido discursivo}

En la Figura 4 se observan los temas centrales o tópicos compartidos por las comunidades, en tanto que, en la Figura 5, se muestra el comportamiento de likes asignados a dichos contenidos compartidos.

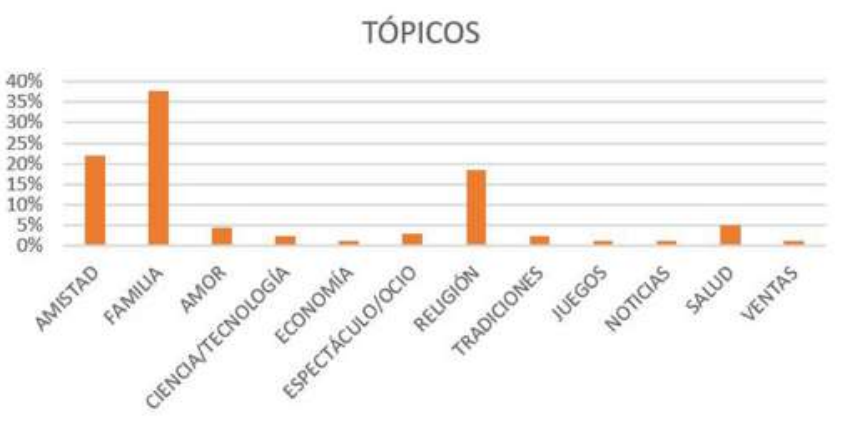

Figura 4. Tópico de contenidos.

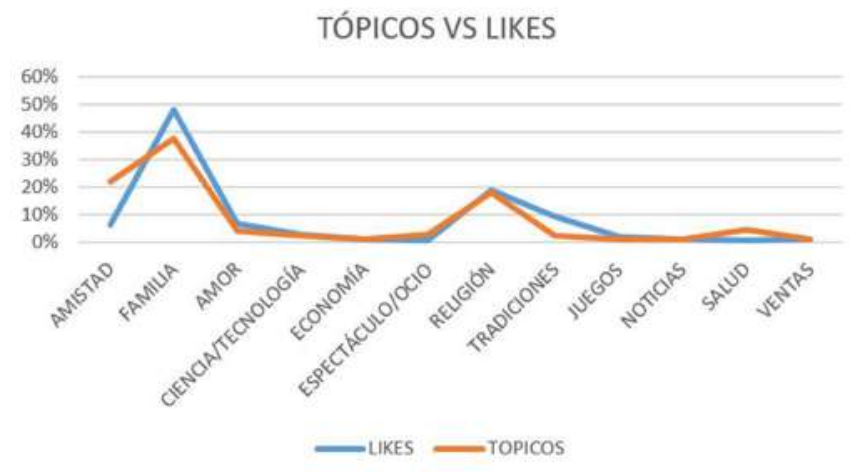

Figura 5. Comparativo entre tópicos utilizados y likes ejercidos por la comunidad. 
Como se desprende de la Tabla 3, el tema de la familia es fundamental para la comunidad, y en segundo lugar la religión. En este segundo punto, la religiosidad es significada como una forma de trascendencia espiritual.

La memoria individual y colectiva está en constante aparición en cada una de las interacciones. La nostalgia es el tamiz de estas memorias.

Áreas como salud, educación, pareja y trabajo, están ausentes de la agenda digital, por lo que la herramienta mantiene este potencial, en tanto que es subutilizada.

Tabla 3. Contenido temático de las diferentes acciones en comunidades virtuales de Facebook.

\begin{tabular}{|c|c|c|}
\hline Acción & Tipo & Index code \\
\hline Comentario & & $\begin{array}{l}\text { Religión } \\
\text { Amistad } \\
\text { Familia }\end{array}$ \\
\hline \multirow[t]{6}{*}{ Share } & Comentario & $\begin{array}{l}\text { Familia } \\
\text { Religión }\end{array}$ \\
\hline & Foto & $\begin{array}{l}\text { Familia } \\
\text { Ventas }\end{array}$ \\
\hline & GIF & $\begin{array}{l}\text { Amistad } \\
\text { Amor } \\
\text { Familia } \\
\text { Religión }\end{array}$ \\
\hline & Imagen & $\begin{array}{l}\text { Amistad } \\
\text { Amor } \\
\text { Ciencia/Tecnología } \\
\text { Economía } \\
\text { Entretenimiento } \\
\text { Salud } \\
\text { Familia } \\
\text { Empleo } \\
\text { Religión } \\
\text { Tradiciones } \\
\text { Juego } \\
\text { Noticias }\end{array}$ \\
\hline & Página web & $\begin{array}{l}\text { Ciencia/Tecnología } \\
\text { Familia } \\
\text { Religión } \\
\text { Salud } \\
\text { Amistad }\end{array}$ \\
\hline & Video & $\begin{array}{l}\text { Amistad } \\
\text { Amor } \\
\text { Ciencia/Tecnología } \\
\text { Ocio/Espectáculo }\end{array}$ \\
\hline
\end{tabular}

Fuente: Elaboración propia.

\subsection{Usuarios de la tecnología}

Este grupo etario procede de entornos familiares extensos y en contacto regular. Generalmente de nivel socioeconómico medio y medio alto $(\mathrm{C}, \mathrm{C}+)$. Por tal motivo, el acceso y familiaridad hacia las tecnologías de información y comunicación es habitual.

La forma en que se utiliza la tecnología es fundamentalmente para cubrir espacios de ocio y como forma de acompañamiento en momentos de soledad, lo cual puede ocupar un rango alto del día (de dos a seis horas). La interacción social es limitada a la familia cercana y son pocos quienes han construido alguna relación afectiva a partir de las redes sociales digitales.

Los usuarios también identifican aspectos negativos que acompañan a los medios sociales o social media, pues, igual que los "no usuarios", resaltan algunos inconvenientes que la acompañan.

\subsection{No usuarios de la tecnología}

Este grupo pertenece a entornos familiares de nivel socioeconómico más bajo. Aunque las causas del no uso de la tecnología son diversas, se pueden asociar en algunos puntos particulares:

a) Incapacidad física: incapacidad asociada al mareo y dolores de cabeza producidos por la exposición a pantalla, problemas visuales y su subsecuente falta de pericia en el uso de la tecnología.

b) Bajo poder adquisitivo: el costo de la tecnología se vuelve inaccesible para un amplio espectro de adultos mayores que ven sus ingresos disminuidos por la jubilación. Esto convierte a las tecnologías como bienes de lujo y quedan supeditados a la intervención financiera de familiares o amigos. Esta relación de dependencia se traduce en algunos casos como violencia económica.

c) Falta de interés: al conceptualizar la tecnología como ajena a su tiempo, pierden el interés por incorporarla en sus hábitos.

d) Analfabetismo tecnológico: la poca interacción con las nuevas tecnologías (interacción disminuida por su desarticulación paulatina con el sistema socioeconómico) genera analfabetismo tecnológico y, 
posteriormente, el abandono de estos instrumentos. Además, se desconocen los usos y alcances de estas herramientas.

Esto refuerza la idea de complejidad de la herramienta tecnológica e induce el miedo por su uso (al ser de costo elevado, algún desperfecto resulta gravoso).

e) Culturales: muchas de estas tecnologías pertenecen a esferas sociales ajenas a muchos grupos sociales, donde los adultos mayores carecen de conocimiento de su existencia.

El discurso asociado a las tecnologías, internet y redes digitales se sintetiza en la Tabla 4.

\section{Cierre de la exploración}

Las experiencias tecnológicas en el mundo de los adultos mayores cuentan con aspectos positivos que deben ser discutidos, no solamente por los desarrolladores de tecnología y académicos, sino justamente con este sector como interlocutor. Aunque varias formas de inclusión surgen al ser considerados poco a poco por los nuevos modelos empresariales en red (Wong, 2013), la incorporación de diferentes tecnologías para reforzar lazos afectivos con miembros de la familia y amistades (Neustaedter, Judge, Hillman, Oduor,\& Pang, 2012) a través de estrategias de comunicación o nuevas formas de socialización hipermediada; representan un reto intergeneracional y una fuente de angustia constante.

En general, las redes sociales son una herramienta de información que en la práctica deja fuera múltiples posibilidades, con esporádicos intentos por explorar otras áreas como son la promoción de cursos y educación para adultos mayores, el autoempleo, talleres especializados, entre muchas otras opciones latentes en la red.

La memoria es fundamental y se vuelve acto al ocupar un lugar central en las relaciones comunitarias que funcionan como espacio ideológico donde se refuerzan las posiciones dentro de narrativas sustentadas a través de imágenes y discursos compartidos y validados por los otros miembros (Giménez, 2009). La red digital, en estos términos, promueve la reactivación de la memoria individual y colectiva en un proceso de comunicación que da coherencia, presencia y sentido de continuidad a los usuarios.
Existe en general una asociación negativa entre la tecnología actual y el uso que se hace de ella en la sociedad. Esta idea es sustentada por discursos mediáticos, principalmente obtenidos en noticieros y comentarios de vox populi.

A diferencia de algunos planteamientos tecnofílicos, los adultos mayores consideran las redes sociales por internet como una pérdida de tiempo valioso para vivir las relaciones interpersonales cara a cara, sector que se ve a sí mismo con poco tiempo para la vida afectiva. Esto promueve el desinterés por el aprendizaje tecnológico y el desconocimiento de las posibilidades de dicha herramienta.

Aunado a lo anterior, también consideran que estos desarrollos tecnológicos, internet y sus diferentes programas, son una manifestación sintomática de las necesidades de esta época, por lo que son desarrollos específicamente generados para los jóvenes y sus necesidades.

Como resultado, la percepción es que estas tecnologías no son de incumbencia ni de interés para los adultos mayores. La vivencia que desprende estas afirmaciones radica en la observación de la tecnología a lo largo de diferentes generaciones, donde han visto una relación análoga entre, por ejemplo, la radio y los abuelos de los entrevistados; la televisión y su propia experiencia juvenil; y en la actualidad, con la computadora y los jóvenes contemporáneos.

El proceso de intrusión tecnológica se hace patente en estas relaciones generacionales con los diferentes desarrollos tecnológicos. En la experiencia personal, la tecnología avanza lentamente en su dimensión técnica, como una herramienta facilitadora en la vida de las personas, incorporándola cada vez más en las relaciones sociales hasta llegar a suplir relaciones afectivas cara a cara.

La radio, la televisión y la computadora -en su condición de medio de comunicación social- siempre han acompañado a las personas en cada época. En un primer momento, la radio sólo acompañaba a su audiencia de manera periférica, impulsando la imaginación por medio del entretenimiento centrado en el oído; la televisión introdujo el valor en la mirada, en detrimento de la imaginación, modificando las relaciones sociales al incidir en la movilidad de su audiencia; por último, la computadora, que se desarrolla en pos de esa movilidad perdida, revalorizándola, y que hoy es central en las 
Tabla 4. Discurso de usuarios versus no usuarios

\begin{tabular}{ll}
\hline \multicolumn{1}{c}{ Usuarios } & \multicolumn{1}{c}{ No usuarios } \\
\hline Sirve para la búsqueda de información & Sirve para la búsqueda de información \\
Esta información es poco fiable & Esta información es poco fiable \\
Es usada de forma negativa & Es usada de forma negativa \\
Es funcional & Es irrelevante \\
Es fundamental para mantener unida a la familia & Lo importante son las relaciones cara a cara \\
Está diseñada para jóvenes & Está diseñada para jóvenes \\
Es costosa & Es costosa \\
Requiere límites y supervisión & Requiere límites y supervisión \\
Su uso en exceso profundiza el aislamiento & Su uso en exceso profundiza el aislamiento \\
Es parte de la cotidianeidad & Es una pérdida de tiempo \\
\hline
\end{tabular}

Fuente: Elaboración propia.

actividades socioeconómicas y culturales. Lo que tienen en común es la función de nodos de transmisión de información que construye la realidad social.

La imagen es la de una tecnología que avanza, que engulle lo que de natural existe en el hombre. En ese sentido, una dialéctica se cierne en los discursos críticos de la modernidad y el papel que juega en ella la tecnología de internet. Esta dialéctica, sintetizada por Carlos Scolari (2008, pp. 145-159), es la pugna entre el progreso como la deshumanización o automatización de la sociedad versus la naturaleza como estado de atraso, analfabetismo y barbarie. Lo viejo es lo natural, lo joven es lo tecnológico. Pero esto implica el avance de una deshumanización que corrompe los valores que están contenidos en el estado natural.

Dentro de estas visiones tecnológicas se encuentra el asilamiento visible en las prácticas sociales comunes que jóvenes y adultos tienen a diario. Idolatría de la máquina que contempla una relación afectiva, no para los cientos de contactos de la red social, sino para el artilugio que hace posible esa red. El artefacto es más importante que su función.

Aun así, la comunidad de adultos mayores reconoce que esta red, como cualquier cosa del mundo, tiene sus aspectos positivos; pero lo son para los otros a quienes se ha destinado sus funciones. Bajo esta perspectiva, estaría el mundo ante el umbral de una resignificación del progreso, pero esta vez nadie lo advierte, sino las generaciones que ya antes lo observaron, durante la primera mitad del siglo pasado y que constituyen voces que se apagan poco a poco.

Por tal motivo, no hay interés por formar parte de ese nuеvo mundo, porque para un adulto mayor el tiempo es más importante si se utiliza en un mundo "natural", donde las relaciones se palpan y se pasan cara a cara. La otra realidad es "artificial", es la pérdida de un valioso tiempo porque, reconocen, las redes sociales deberían ser más importantes por su densidad afectiva que por la cantidad de "amigos" que las componen.

\section{Referencias}

DeWalt, K., DeWalt, B. (2011) Participant Observation. A guide for fieldworkers Second Edition. United Kingdom: AltaMira Press.

Galindo Cáceres, J. (2000) Construcción de una comunidad virtual. La red-sistema de formación en altos estudios de la red de comunicación compleja. Signo y Pensamiento, vol. XIX, 36, 2000, pp. 93-102 Pontificia Universidad Javeriana Bogotá, Colombia.

Galindo Cáceres, J. (1992) Ideología y comunicación. El Estado, la Hegemonía y la Difusión masiva, Premiá, México.

Giménez, G. (2009) "Cultura, identidad y memoria. Materiales para una sociología de los procesos culturales en las franjas fronterizas" Frontera norte vol.21, 41 México ene./jun. 2009.

Hammersley, M., Atkinson, P. (1995) Etnografía. Método de investigación. Barcelona. Paidos.

Hine, C. (2004) Etnografía virtual. Colección Nuevas Tecnologías y Sociedad. Editorial UOC. Barcelona, España.

INEGI (2010). Censo de Población y Vivienda 2010.

INEGI (2017a) Estadísticas a propósito del día mundial de internet (17 de mayo). Datos nacionales. Aguascalientes, Ags. 
INEGI (2017b) Encuesta Nacional sobre Disponibilidad y Uso de Tecnologías de la Información en los Hogares (ENDUTIH), 2017.

Llorente, C., Viñarás, M., y Sánchez Valle, M. (2015) Mayores e Internet: La Red como fuente de oportunidades para un envejecimiento activo. Comunicar, 45, v. XXIII, 2015

Neustaedter, C., Judge, T. K., Hillman, S., Oduor, E. \& Pang, C. (2012) Connecting Families through Technology, Proceedings of ACM CHI 2012 Workshop on Technologies for Today's Families.

OMS (s.f.) Envejecimiento y ciclo de vida. http://www.who. int/ageing/about/facts/es/ recuperado el 19 de mayo de 2016.

Schützeichel, R. (2015) Teorías sociológicas de la comunicación. Universidad Iberoamericana. Colección Teoría Social. México.

Scolari, C. (2008) Hipermediaciones. Elementos para una teoría de la comunicación digital. Ed. Gedisa. Barcelona.

Spradley, J. (1980) Participant Observation. New York. Holt, Rinehart and Winston.

Villafranco, G. (2015) Los estados con mayor crecimiento económico en México Forbes México, febrero 11, 2015. Recuperado el 29 de julio de 2018 https://www.forbes.com.mx/los-estados-con-mayor-cr ecimiento-economico-en-mexico/

Wong, C. B. (2013) Perceived Usefulness of Internet for Adopters and Non-adopters: An Empirical Analysis of Retired Senior Citizens in Hong Kong. GSTF Journal on Computing Vol 2 Núm. 4. Hong Kong. 